\title{
Preparation and Properties of Degradable Metal Materials for Oil and Gas Wells
}

\author{
Zhang Yi ${ }^{1}$, Liu Jinhu ${ }^{2}$, Shi Xiaolong ${ }^{1}$, Chang Gang $^{3}$, Liu Wentao ${ }^{4}$, Nie Wei ${ }^{1}$, Yang Dong ${ }^{1}$ \\ ${ }^{1}$ Downhole Service Company, BHDC, CNPC Renqiu, Hebei, China \\ ${ }^{2}$ Jiangsu Xuzhou Mining Group Co., Ltd Xuzhou, Jiangsu, China \\ ${ }^{3}$ Staff Education And Training Center, Bhdc, Cnpc Dagang, Tianjin, China \\ ${ }^{4}$ Tubing Service Company, Bhdc, Cnpc Renqiu, Hebei, China
}

\section{Email address:}

zhangyi_zy@cnpc.com.cn (Zhang Yi),362105376@qq.com (Liu Jinhu),785671750@qq.com (Shi Xiaolong),

54976875@qq.com (Chang Gang), liuwentaowy@163.com (Liu Wentao), nie_wei@cnpc.com.cn (Nie Wei), yangdong2000@cnpc.com.cn (Yang Dong)

\section{To cite this article:}

Zhang Yi, Liu Jinhu, Shi Xiaolong, Chang Gang, Liu Wentao, Nie Wei, Yang Dong. Preparation and Properties of Degradable Metal Materials for Oil and Gas Wells. Science Discovery. Vol. 8, No. 2, 2020, pp. 37-42. doi: 10.11648/j.sd.20200802.13

Received: February 3, 2020; Accepted: May 20, 2020; Published: June 9, 2020

\begin{abstract}
In the process of measure operation for oil and gas wells, downhole tools may get stuck, which not only delays the construction period, but also increases the operation cost. This paper discusses a kind of degradable metal material which can be used to make downhole tools for oil and gas wells. In order to prepare the material, firstly, according to the requirements of downhole operation technology of oil and gas wells, the overall design idea with magnesium and magnesium alloy as the core was determined, and then the $\mathrm{Ni}, \mathrm{Ti}, \mathrm{Cr}, \mathrm{Cu}$ and other metals were determined as auxiliary materials to enhance the alloy strength through electrochemical experiments. After that, these materials are made into degradable metal bars which can be processed by high-temperature and high-pressure die-casting. At last, they are verified by metallographic characterization, tensile impact mechanics experiment, microhardness test, degradation performance and other experiments, which can meet the design and degradation requirements of some downhole tools with low strength requirements. This kind of degradable metal material has the characteristics of light weight, high strength and easy flowback after construction. It is suggested that it should be widely used in downhole tools such as perforating gun, packer, bridge plug and fracturing sliding sleeve.
\end{abstract}

Keywords: Degradable, Magnesium Alloy, Electrochemical Corrosion, Pressure Casting

\section{油气井用可降解金属材料制备及性能研究}

\author{
张毅 ${ }^{1}$, 刘金虎 ${ }^{2}$, 石小龙 ${ }^{1}$, 常刚 ${ }^{3}$, 刘文韬 ${ }^{4}$, 聂伟 $^{1}$, 杨东 ${ }^{1}$ \\ ${ }^{1}$ 中国石油集团渤海钻探井下作业分公司, 任丘, 河北, 中国 \\ 2江苏省徐州矿务集团有限公司, 徐州, 江苏, 中国 \\ ${ }^{3}$ 中国石油集团渤海钻探培训中心, 大港, 天津, 中国 \\ ${ }^{4}$ 中国石油集团渤海钻管具技术服务分公司，任丘，河北，中国
}

\section{邮箱}

zhangyi_zy@cnpc.com.cn（张毅），362105376@qq.com（刘金虎）, 785671750@qq.com(石小龙）, 54976875@qq.com（常刚）, liuwentaowy@163.com (刘文韬), nie_wei@cnpc.com.cn（聂伟），yangdong2000@cnpc.com.cn（杨东）

摘要：对油气井进行措施作业过程中可能会发生井下工具遇卡事故，不仅耽误施工周期，还会增加作业成本。本文所 探讨的是一种可用于井下工具制作的油气井用可降解金属材料。为制备该材料首先根据油气井井下措施作业工艺要求, 确定了以镁及镁合金为核心的总体设计思路，再通过电化学实验确定了 $\mathrm{Ni} 、 \mathrm{Ti} 、 \mathrm{Cr} 、 \mathrm{Cu}$ 等金属作为增强合金强度的辅 
料材料。之后将这些材料进行高温高压压铸成型, 制作成可以进行零件加工的可降解金属棒材, 最后经金相表征、拉 伸冲击力学实验、显微硬度测试、降解性能等实验验证, 可以满足一些对强度要求不是太高的井下工具的设计以及降 解要求。该制备的可降解金属材料具有质量轻、强度相对较高、施工完毕后易返排等特点, 建议可在射孔枪、封隔器、 桥塞以及压裂滑套等井下工具上进行推广应用。

关键词：可降解, 镁合金, 电化学腐蚀, 压铸

\section{1. 引言}

对油气井进行措施作业过程中, 经常会出现因地层高 温、出砂、管外萓、井口落物、工具性能不稳定等发生的 井下工具遇卡事故, 这时需要进行打捞、钻磨等处理, 处 理过程中修井液以及钻磨钻屑会不仅对储层造成伤害, 也 耽误了工时, 增加了作业成本。试想在工艺条件允许的情 况下, 使用可降解金属材料制作井下工具, 如发生遇卡, 此时只需注入降解液或者在井内产出高氯根水溶液影响 下工具便可自行降解, 经洗井返排后提出管柱即可, 大大 降低了工具遇卡事故发生的风险。

油气井用可降解金属材料的研究最早始于斯伦贝谢、 贝克休斯等几家跨国油服公司。其中, 斯伦贝谢公司的可 降解镁铝合金材料具有耐冲蚀、降解速率可控、无需化学 添加剂和适于低 $\mathrm{PH}$ 值环境等优点, 适用于不同的井深、 温度、压力和流体特性等条件, 经实验, 该类合金工具的 最大工作压差可达 $70 \mathrm{MPa}$, 无需回收或钻磨作业, 其降解 的副产品为微米级粉末, 不影响返排和油气生产。由贝克 休斯公司研发的可降解纳米复合材料, 主要由活性金属基 质和增强部分组成, 采用粉末冶金工艺固化活性金属粉末 并在表面覆盖金属或陶瓷以增强硬度，具有低密度、高强 度、降解速率可控和低成本的特点, 抗压强度高达 $830 \mathrm{MPa}$, 破裂伸长率为 $8 \% \sim 12 \%$ 。我国对可降解金属材料的研究起 步较晚, 但也取得了部分成果, 国内中国石油勘探开发研 究院裴晓含应用可降解金属材料研制了适用于多级投球 滑套分段压裂的可分解压裂球, 分析了可分解压裂球材料 的分解特性及力学性能, 并对可分解压裂球进行了地面承 压试验及现场应用。此外, 中石化石油机械股份有限公司 周歆通过对可降解金属材料从承压性能、降解性能的各种 测试中分析获得了的降解速率预测公式, 为现场应用提供 技术指导并现场应用成功[1]。

\section{2. 可降解金属材料设计思路}

井下工具多用于油气井分层措施作业中, 在使用过程 中不仅需要保证强度满足施工条件, 而且施工完成后如果 遇卡在注入降解液或者井内产出高氯根水溶液的情况下 工具可自行降解 [2], 所以在选材料方面, 其设计应达到以 下技术要求:

1) 该种材料为复合组合材料;

2) 一定的耐温性, 满足耐温 $150^{\circ} \mathrm{C}$ 以上, 高的抗拉强度, 满足300MPa以上，合适的密度，低于 $2.3 \mathrm{~g} / \mathrm{cm} 3$;

3) 良好可加工性, 便于加工成各种规格、各种形状的金 属棒;
4) 匹配的电偶腐蚀性能, 满足电解质溶液中材料的降解 要求;

$5)$ 温度在 $150^{\circ} \mathrm{C}$ 内，在 $5 \% \mathrm{KCl}$ 溶液中，可降解金属材料 降解速度要控制在大于 1 天小于 5 天。

综合考虑, 目前最适合可降解金属材料的主体核心为 镁及镁合金。镁及镁合金是工程应用中密度最低的金属材 料, 具有低密度、高比强度和比刚度, 减振性好、电磁屏 蔽效果佳和抗福射能力强、资源丰富, 环境友好等优点, 在航空航天、交通、汽车、电子仪器等国防工业和民用工 业中的应用前景非常广阔。随着镁合金材料科学技术的深 入研究, 其性能特点不断被挖掘和应用, 已经发展成为最 轻的金属结构材料。与铸造镁合金相比, 变形镁合金具有 力学性能好、强度高、塑性好、组织致密, 性能稳定、规 格多样化等特点, 除此之外, 镁合金在高氯根的水溶液里 容易发生电化学腐蚀, 降解性能良好, 适合于制作可降解 金属材料[3]。

\section{3. 可降解金属材料的电化学实验}

镁及镁合金虽然具有诸多有优点, 但强度较低, 需添 加提高强度的金属材料。可提高合金强度的金属有许多, 在综合考虑多种可降解金属材料的电化学腐蚀性能前提 下, 制备了NiTi、TiAl、Ti ${ }_{3} \mathrm{Al} 、 \mathrm{CuAl} 、 \mathrm{NiCu} 、 \mathrm{NiCr} 、 \mathrm{NiAl}$ 、 $\mathrm{Ni}$ 多种合金材料。表1是设计的不同复合材料组成[4]。

表1 可溶解金属棒复合粉体材料组成。

\begin{tabular}{ll}
\hline 材料 & 组成 \\
\hline $\mathrm{NiTi}$ & 摩尔比 $1: 1$ \\
$\mathrm{TiAl}$ & 摩尔比 $1: 1$ \\
$\mathrm{Ti}_{3} \mathrm{Al}$ & 摩尔比 $3: 1$ \\
$\mathrm{CuAl}$ & 摩尔比 $1: 1$ \\
$\mathrm{NiCu}$ & 质量比 $4: 1$ \\
$\mathrm{NiCr}$ & 质量比 $4: 1$ \\
$\mathrm{NiAl}$ & 质量比 $4: 1$ \\
$\mathrm{Ni}$ & $100 \%$ \\
\hline
\end{tabular}

对这些材料进行电化学测试, 电化学测试主要测试了 制备的各种复合粉体材料的开路电位、动电位极化曲线等。 金属(或合金)在电解液中所产生的电极电位, 称为该金属 (或合金)在该溶液中的开路电位, 这是衡量其电化学性能 的重要指标 [5]。

实验采用三电极体系, 以粉体压制烧结的合金试样为 研究电极, 以铂电极为辅助电极, 以饱和甘录电极 (SCE) 为参比电极。试验中将试样切割成面积为 $1 \mathrm{~cm} 2$ 的试片。 测试溶液为 $5 \% \mathrm{NaCl}$ 溶液, 实验温度控制在 $35 \pm 2^{\circ} \mathrm{C}$ 。测试 前向溶液中通入氧气至少 $20 \mathrm{~min}$ 获得饱和氧溶液测试时 间为 $180 \mathrm{~min}$ 。待试样开路电位稳定后测试合金试样的动 
电位极化曲线, 扫描速率为 $0.166 \mathrm{mV} / \mathrm{s}$, 测试结果如图 1 , 图2所示。

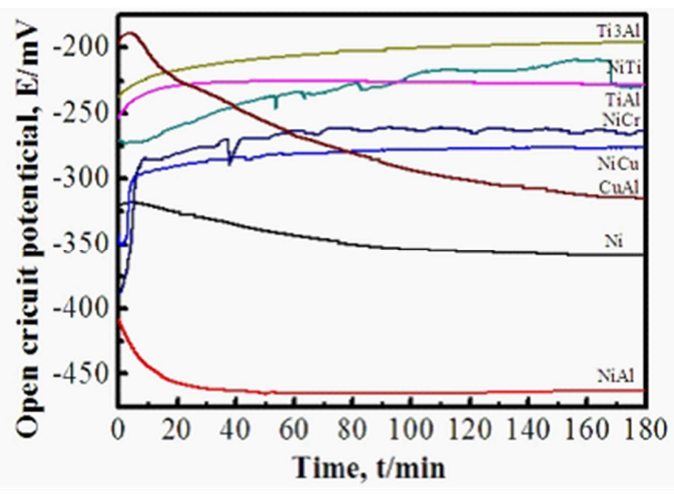

图1 不同可溶解金属棒复合材料的腐蚀电位。

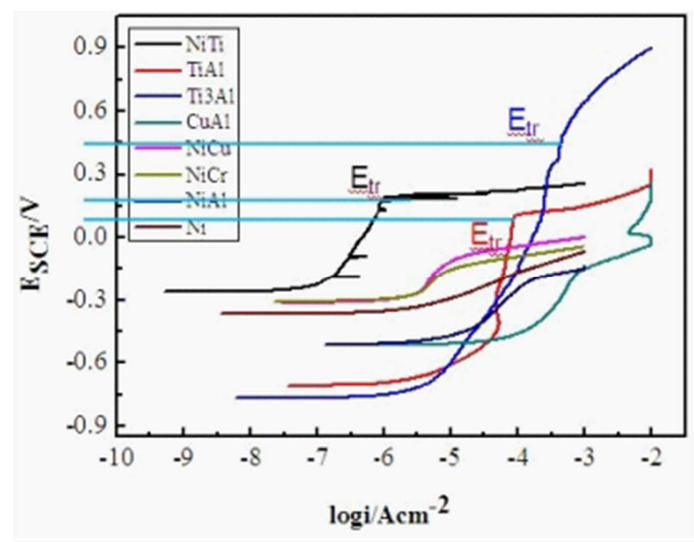

图2 不同可溶解金属棒复合材料的阳极极化曲线。

图1是不同可降解金属棒复合材料的腐蚀电位。从图 中可以看出, $\mathrm{NiTi} 、 \mathrm{TiAl} 、 \mathrm{Ti}_{3} \mathrm{Al} 、 \mathrm{NiCr}$ 有着较高的腐蚀电 位, 耐 $\mathrm{Cl}^{-}$腐蚀性较好。图2是不同可溶解金属棒复合材料 的阳极极化曲线。可以发现, $\mathrm{NiTi} 、 \mathrm{TiAl} 、 \mathrm{Ti}_{3} \mathrm{Al}$ 出现了明 显的钝化现象。其中 $\mathrm{Ti}_{3} \mathrm{Al}$ 有较宽的钝化区间, 随着电位的 增加未出现电流密度陡然增大的现象, 说明未发生局部腐 蚀。TiAl在Etr处被击穿, 发生点蚀。NiTi在阳极扫描的过 程中多次发生击穿、自愈合, 电位继续增大, 在Etr处电流 陡然增大, 发生了大规模的局部腐蚀, 但此处电位高达 $0.2 \mathrm{~V}$, 所以在 $\mathrm{Cl}^{-}$存在条件下抗腐蚀性能较好。 $\mathrm{CuAl} 、 \mathrm{NiCu}$ 、 $\mathrm{NiCr} 、 \mathrm{NiAl} 、 \mathrm{Ni}$ 材料钝化区间小，相对来说耐 $\mathrm{Cl}^{-}$腐蚀性能 要稍微差一些[6]。

综合以上的电化学实验数据, 我们拟选择的可溶解金 属棒材料的主体材料为镁及其合金材料, 通过添加 $\mathrm{Ni} 、 \mathrm{Ti}$ 、 $\mathrm{Cr} 、 \mathrm{Cu}$ 等金属元素等进一步提高其强度, 同时降低其耐电 解质腐蚀性能。

\section{4. 可降解金属棒材制备}

可降解工具各零件的加工是在制备成型的可降解金 属棒材的基础上进行车削, 可降解金属棒材制备采用的是 压铸生产, 压铸生产的基本原理是将液态或半液态金属在 高压作用下, 以较高速度充填到模具中, 并在压力作用下 凝固而获得所需铸件。次辞制备采用的是200公斤坩埚电 阻炉进行熔炼, 压铸机为压力1600吨卧室冷室压铸机[7]。 制备的试样如图3所示。

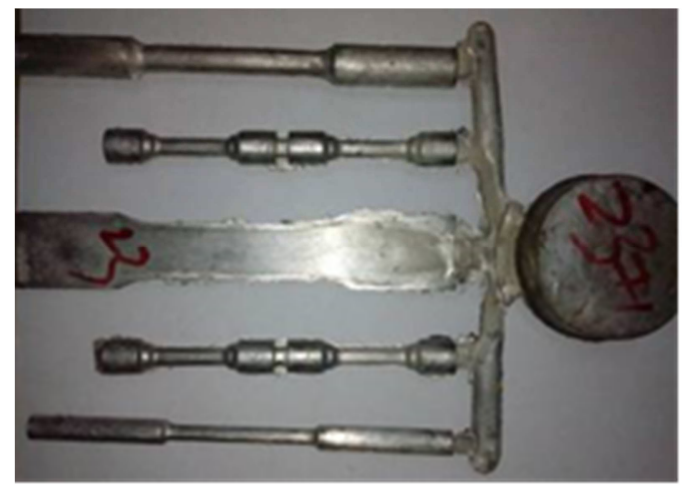

图3 压铸件实物图。

\section{5. 实验}

\section{1. 室温拉伸力学性能}

本试验采用电子万能材料试验机进行压铸镁合金的 力学性能测试。制定试样时, 车削成如图4所示尺寸的标 准拉伸试样。

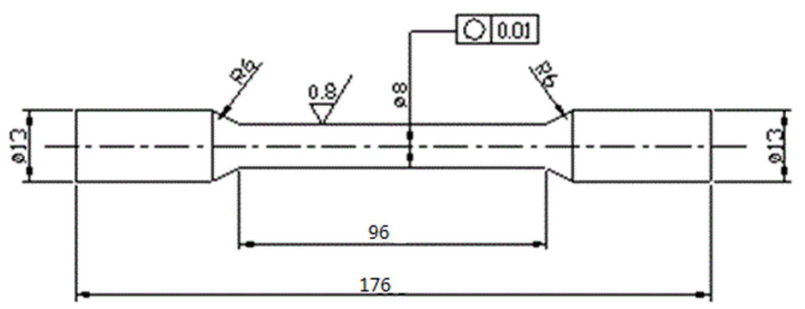

图4 拉伸试样尺寸示意图。

力学性能测试过程中, 采用匀速、单向拉伸, 单向拉 伸速率设置为 $3 \mathrm{~mm} / \mathrm{min}$ 。为了保证拉伸力学性能的准确度, 准备2个试样进行拉伸试验。抗拉强度355-381MPa, 屈服 强度270-350MPa, 延伸率为6.5-7\%, 如表2所示, 满足一 般铝合金的强度水平。

表2 样品力学性能。

\begin{tabular}{llll}
\hline \multirow{2}{*}{ 样品编号 } & 抗拉强度 & 规定塑性延伸强度Rp0.2/MPa & 断后伸长率 $\mathbf{A} \%$ \\
\hline 1 & $\mathbf{R m} / \mathbf{M P a}$ & 270 & 6.5 \\
2 & 355 & 350 & 7 \\
\hline
\end{tabular}

采用夏比冲击摆锤进行能量吸收试验, 使用深度为 $2 \mathrm{~mm}$ 的U型缺口试样, 冲击吸收能量为1.1-1.2焦耳, 如表3所示, 说明该材料韧性较差。 
表3 样品冲击测试。

\begin{tabular}{llll}
\hline 样品编号 & 实验温度 ${ }^{\circ} \mathbf{C}$ & 冲击吸收能量 $\mathbf{K U}_{2} / \mathbf{J}$ & 备注 \\
\hline 1 & 室温 & 1.2 & 吸收能低于试验机最小分辨力的 2.5 倍 \\
2 & 室温 & 1.1 & 吸收能低于试验机最小分辨力的 2.5 倍 \\
\hline
\end{tabular}

\section{2. 显微硬度}

试验选用Microhardness Tester HV-1000型显微硬度计 进行显微硬度的测试。试样经砂纸打磨后, 用压平器嵌入 载物台, 保证测试面在同一水平线上。试验选取载荷为
$100 \mathrm{~g}$, 保载时间为 $15 \mathrm{~s}$ 。试验过程中应使测试仪的压头作 用在基体的晶粒内部, 避免打在晶界上。选取中心到边缘 均匀分布 10 点进行压点试验 [8]。并绘制如图5所示的合金 硬度折线图。

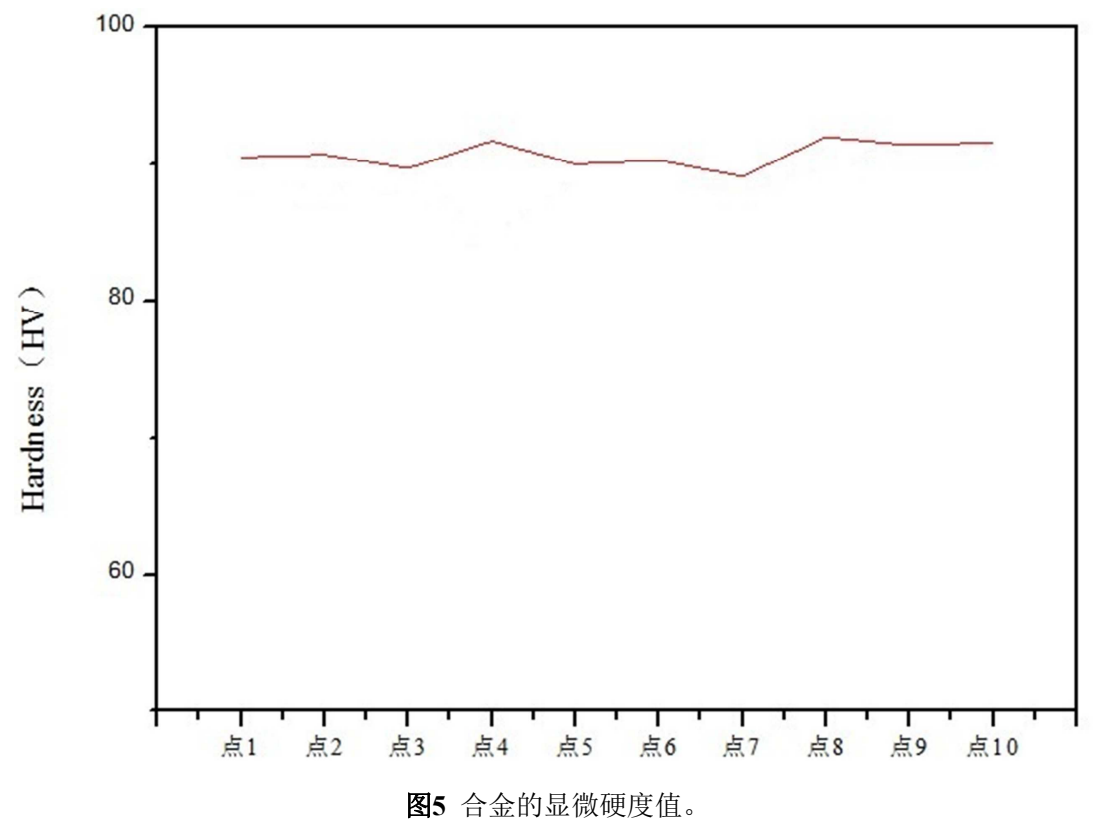

由此可以得出该合金 10 个点的平均洛氏硬度为 94.8 , 满足一般铝合金的硬度水平。

\section{3. 低倍组织观察}

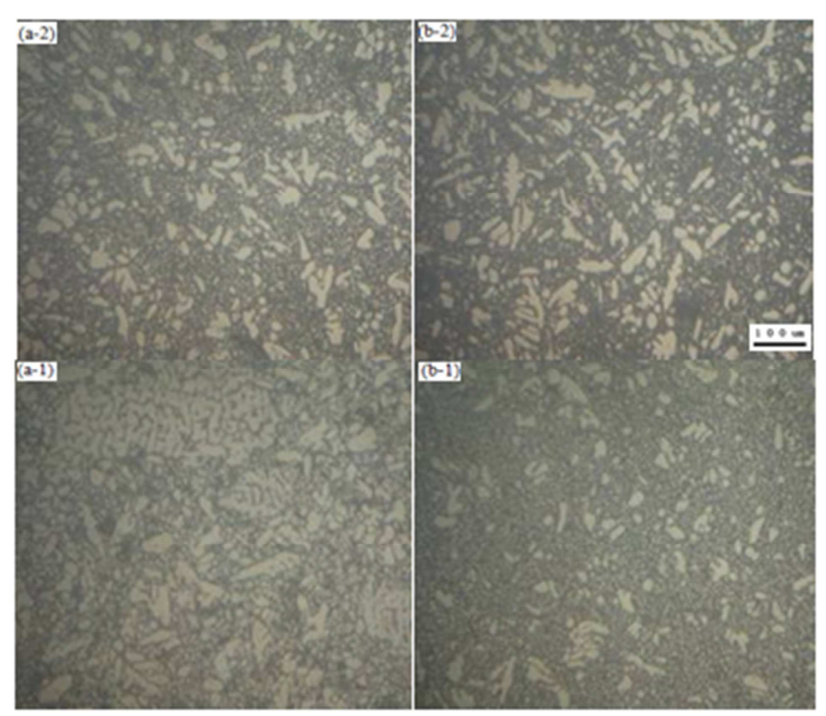

图6 压铸后的金相组织照片。

采用400\#水磨砂纸粗磨再改用600\#砂纸、800\#砂纸和 1200\#砂纸精磨, 磨面在达到要求后, 使用酒精清洗吹干。
用佳能DS126181数码相机摄取获得了样品100-1000倍的 金相组织结构, 如图6所示: 样品金相组织为黑灰色+白色 组织, 低倍下组织均匀, 晶粒细小。高倍下观察黑灰色组 织, 主要有类似珠光体的片层状和团絮状两种形貌, 推测 为 $\mathrm{Mg}$ 和 $\mathrm{A} 1$ 或其它合金元素的共晶相, 灰色相与黑色相可 能是不同的共晶组织。白色组织在高倍下可见清晰的晶界, 且在晶界处有少量粒状析出相, 推测为 $\mathrm{Mg}$ 合金 $\mathrm{a}$ 相 [9]。如 图6的金相组织的照片。

\section{4. 降解实验}

采用恒温水浴浸泡的方法测试可降解材料在不同条 件下的降解速率。将选取的可降解镁铝合金材料按照封隔 器刚体比例缩小, 加工成圆筒状实验样品, 进行了 $50^{\circ} \mathrm{C}$ 、 $70^{\circ} \mathrm{C}$ 和 $90^{\circ} \mathrm{C}$ 温度下降解性能试验, 以及浸泡在浓度为 $1 \%$ 、 $2 \% 、 5 \%$ 的 KCl溶液中的盐水降解试验。每隔 $2 \mathrm{~h}$ 烘干样品, 采用精密电子天平称取试样剩余质量, 直至试样完全降解 [10]。

\subsection{1. 温度对材料降解性能的影响}

试验了 $50^{\circ} \mathrm{C} 、 70^{\circ} \mathrm{C}$ 和 $90^{\circ} \mathrm{C}$ 不同温度条件下, 在浓度为 $5 \%$ 的 $\mathrm{KCl}$ 溶液中, 可降解材料试样浸泡后剩余质量, 如表 4, 并绘制剩余质量与溶解时间的关系曲线如图7所示。 
表4 5\%的KCl溶液中, 不同温度下的降解。

\begin{tabular}{lllllllll}
\hline 质量 & $\mathbf{0 h}$ & $\mathbf{2 h}$ & $\mathbf{6 h}$ & $\mathbf{1 0 h}$ & $\mathbf{1 3 h}$ & $\mathbf{1 6 h}$ & $\mathbf{2 0 h}$ & $\mathbf{2 4 h}$ \\
\hline $50^{\circ} \mathrm{C}$ & 13.5 & 13.5 & 13.5 & 13.46 & 13.4 & 13.35 & 13.25 & 13.11 \\
$70^{\circ} \mathrm{C}$ & 13.5 & 13.5 & 13.49 & 13.41 & 13.31 & 13.2 & 13.01 & 12.64 \\
$90^{\circ} \mathrm{C}$ & 13.51 & 13.51 & 13.47 & 13.29 & 13.01 & 12.72 & 12.01 & 11.06 \\
\hline & & & & & & & & \\
\hline
\end{tabular}

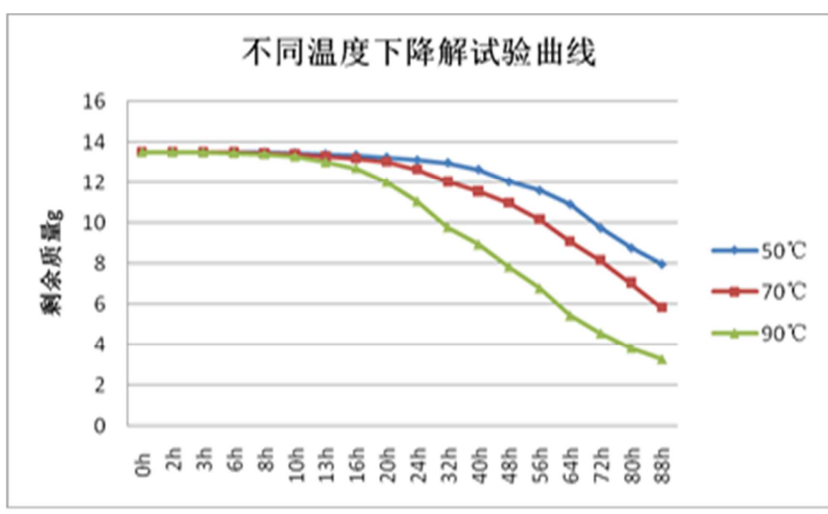

图7 不同温度条件下 $5 \%$ 浓度的KCL溶液中降解试验曲线。

可以看出, 试样在 $50^{\circ} \mathrm{C}$ 的 $5 \%$ 浓度的 $\mathrm{KCl}$ 溶液中前 $10 \mathrm{~h}$ 浸泡后金属表面开始出现腐蚀现象,经过 $16 \mathrm{~h}$ 浸泡后, 金属 表面变化明显, 但尺寸, 质量基本没有变化,经过浸泡 $24 \mathrm{~h}$ 后, 金属表面的腐蚀层有部分脱落现象, 有黑色颗粒掉下, 且质量变化开始减少。经过 $48 \mathrm{~h}$ 浸泡后, 腐蚀表面凹坑明 显增多, 腐蚀深度增大, 质量变化明显减小, 表面出现糊 状模糊的腐蚀, 直至完全溶解[11]。
对于在 $70^{\circ} \mathrm{C}$ 的 $5 \%$ 浓度的 $\mathrm{KCl}$ 溶液中浸泡的试样, $10 \mathrm{~h}$ 之内质量无明显变化, $24 \mathrm{~h}$ 之后质量迅速减少; 对于在 $90^{\circ} \mathrm{C}$ 的 $5 \% \mathrm{KCl}$ 溶液中浸泡的试样, 质量在浸泡后13h后就开始 明显减少， $88 \mathrm{~h}$ 失重 $76 \%$ 。

\subsection{2. 盐水浓度对材料降解性能的影响}

将试样分别浸泡在 $1 \% 、 2 \% 、 5 \% \mathrm{KCL}$ 溶液中，加温至 $90^{\circ} \mathrm{C}$, 每 2 小时测量试样浸泡后剩余质量, 如表 5 , 绘制剩 余质量与溶解时间的关系曲线如图8所示。

\section{材料在不同浓度盐水中的降解}

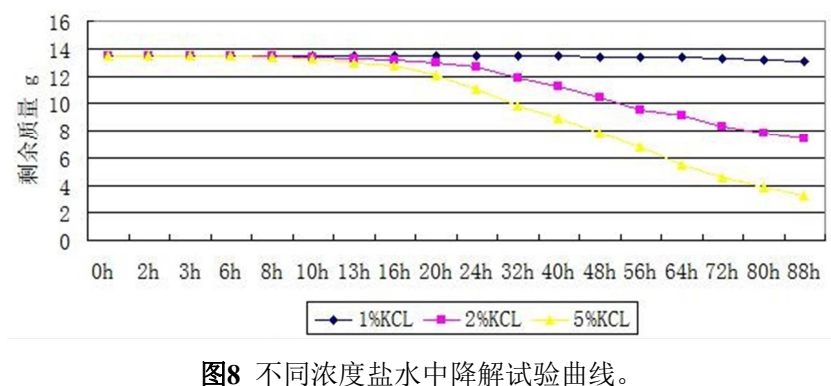

图8 不同浓度盐水中降解试验曲线。

表5 不同浓度盐水中的降解。

\begin{tabular}{lllllllll}
\hline 质量 & $\mathbf{0 h}$ & $\mathbf{6 h}$ & $\mathbf{1 0 h}$ & $\mathbf{1 6 h}$ & $\mathbf{2 4 h}$ & $\mathbf{4 0 h}$ & $\mathbf{5 6 h}$ & $\mathbf{7 2 h}$ \\
\hline $1 \% \mathrm{KCL}$ & 13.45 & 13.45 & 13.45 & 13.45 & 13.44 & 13.44 & 13.39 & 13.25 \\
$2 \% \mathrm{KCL}$ & 13.5 & 13.49 & 13.41 & 13.2 & 12.64 & 11.24 & 9.54 & 8.35 \\
$5 \% \mathrm{KCL}$ & 13.51 & 13.47 & 13.29 & 12.72 & 11.06 & 8.94 & 6.79 & 4.58 \\
\hline
\end{tabular}

试样在 $90^{\circ} \mathrm{C}$ 的 $1 \% \mathrm{KCL}$ 中, $48 \mathrm{~h}$ 金属表面表面开始出现 腐蚀现象, 质量基本没有变化, $72 \mathrm{~h}$ 保持基本形状, 质量开 始有变化; 试样在 $90{ }^{\circ} \mathrm{C}$ 的 $2 \% \mathrm{KCL}$ 中， $6 \mathrm{~h}$ 金属表面表面开 始出现腐蚀现象, 质量基本没有变化, 16h质量变化明显, $88 \mathrm{~h}$ 失重 $45 \%$; 在 $90^{\circ} \mathrm{C}$ 的 $5 \% \mathrm{KCl}$ 溶液中浸泡的试样, 质量 在浸泡后 $10 \mathrm{~h}$ 内就开始明显减少, $88 \mathrm{~h}$ 降解为碎片, 明显快 于 $1 \% \mathrm{KCL}$ 以及 $2 \% \mathrm{KCL}$ 中降解速度 $[12]$ 。

\section{6. 结论与认识}

1) 选择镁铝基金属体系作为可降解金属材料的基底, 再 通过压铸工艺添加 $\mathrm{Ni} 、 \mathrm{Ti} 、 \mathrm{Cr} 、 \mathrm{Cu}$ 等金属，通过特 定的后续热处理工艺对其组织结构进行优化, 获得了 具有强度高、耐冲蚀、降解速率可控的可降解金属材 料。经金相表征、力学参数、降解性能等实验验证该 可降解材料的强度达到一般铝合金水平, 满足一些对 强度要求不是太高的井下工具的设计;
2) 基于可降解材料具有质量轻、强度高、施工完毕后易 返排等特点, 建议在射孔枪、封隔器[13]、桥塞以及 压裂滑套等工具上进行推广应用;

3) 该材料在较高氯根的水溶液中降解速率难以把控, 建 议使用该材料制作的井下工具前应适当降低井内氯 根含量或者在金属表面涂抹一层黄油以减缓降解速 率。此外为满足施工工艺的前提下尽量采用连续油管 进行作业以保证工具的可靠性[14]。

\section{参考文献}

[1] 董明键,郭先敏,李子良.可降解材料在完井工具中的应用及 发展趋势[J].石油机械,2015,43(03):31-34.

[2] Aviles I, Marya M, Hernandez T R, et al. Application and benefits of degradable technology in open-hole fracturing [R]. SPE 166528, 2013. 
[3] Salinas B J, Xu Zhiyue, Agrawal G, et al. Controlled electrolytic metallics-an interventionless nanostructured platform [R]. SPE 153428, 2012.

[4] 裴晓含,魏松波,石白茹,等. 投球滑套分段压裂用可分解压 裂球 [J].石油勘探与开发,2014, 41(06):738-741.

[5] 周歆,杨小城. 可降解压裂球试验研究及现场应用[J].石油矿 场机械,2018,47(01):62-66.

[6] 王林,张世林,平恩顺,等.分段压裂用可降解桥塞研制及其性 能评价[J].科学技术与工程,2017,17(24):228-232.

[7] 张毅,李景卫,杨小涛,等.新型可降解压裂封隔器胶筒[J].油 气井测试,2019,28(02):94-97.

[8] 张毅,刘晓林,王长恩,等. 可降解材料的性能表征及在压裂 领域中的应用 [J].新疆石油天然气, 2019,15(03):51-55.

[9] 朱晓蓉,吴雷泽,代理震.封隔器设计基础[M]. 北京:中国石化 出版社,2012,4:48-52.

[10] 李高升. 封隔器卡瓦的强度分析[D]. 中国石油大学,2007.

[11] 万小勇,李林涛,黄传艳, 等.可取式液压封隔器耐高温胶筒优 选与试验研究 [J].石油机械,2018,46(12):88-93

[12] 江华.影响隔离封隔器坐封深度的因素与确定方法探讨 [J]. 广东化工,2018,45(11):219-220.

[13] 刘江浩,张毅. 高温高压大通径压裂封隔器的研制 [J]. 钻采 工艺,2016,39(03):77-79.

[14] 张毅,于丽敏,任勇强,等.一种新型可降解压裂封隔器坐封球 [J].油气井测试,2018,27(02):53-58.

\section{作者简介}

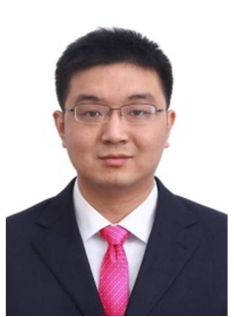

具中心。
张毅, 男, 1987年6月出生, 工程硕士, 工程师，2017年毕业于中国石油大学(华 东)石油与天然气专业, 目前工作于: 中 国石油集团渤海钻工程有限公司探井下 作业分公司, 主要从事油气田试油、试 气技术管理工作。通信地址: 河北省任 丘市会战道渤海钻探井下作业分公司管 